\title{
Fulminant Nocardia Colitis: A Case Report
}

\author{
David Turer, Brian Gray, and Krishnan Raghavendran
}

\begin{abstract}
Background: Nocardia infection is a rare but well-recognized side effect of immunosuppressive therapy including tumor necrosis factor- $\alpha$ (TNF- $\alpha)$ antagonists. However, Nocardia colitis has only been previously reported once in the English literature.

Case Report: We present a 58-y-old female with history of Crohn disease maintained on multiple medications including infliximab (a TNF- $\alpha$ antagonist). The patient presented to the emergency department with hematochezia and abdominal pain. Computed tomography (CT) scan revealed extensive pneumotosis throughout the bowel as well as mesenteric and portal venous gas. She underwent an exploratory laparotomy that revealed bowel edema without ischemia. No bowel was resected and at a planned second-look laparotomy $48 \mathrm{~h}$ later, the bowel appeared normal and the abdomen was closed. Post-operatively, the patient tested negative for Clostridium difficile infection. The blood and abdominal fluid cultures were positive for Nocardia farcinica.

Conclusion: The patient had a complicated post-operative course and developed multiple hospital-acquired infections. However, she survived and was placed in sub-acute rehabilitation four weeks after the acute surgical intervention.
\end{abstract}

$\mathbf{W}$ E PRESENT THE case of a patient with Nocardia colitis and disseminated nocardiosis. The 58-y-old female patient had a past medical history significant for Crohn disease that had required multiple small bowel resections in the distant past. At presentation, she was being maintained on azathioprine, mesalamine, budesonide, and infliximab, a tumor necrosis factor- $\alpha$ (TNF- $\alpha)$ antagonist. Four weeks before presentation the patient had been hospitalized for community-acquired pneumonia and was treated with a course of intravenous antibiotics before being discharged home with a course of oral antibiotics. After discharge, the patient had several weeks of mild abdominal pain and underwent an esophagogastroduodenoscopy (EGD) as an outpatient that revealed no abnormalities. She subsequently presented to the emergency department with hematochezia and moderate bilateral lower quadrant abdominal pain. Computed tomography (CT) scan performed at this time re- vealed extensive pneumotosis in the wall of the distal small bowel and entire colon as well as mesenteric and portal venous gas (Fig. 1).

A detailed laboratory evaluation revealed a significant leukocytosis, lactic acidosis, and evidence of acute kidney injury. After appropriate resuscitation, she was taken emergently to the operating room for exploratory laparotomy. Intra-operatively, she was found to have to have mild small bowel dilation and mild edema of the colon but no evidence of transmural ischemia or inflammation. A flexible sigmoidoscopy was performed that revealed multiple areas of ulceration and membranes consistent with pseudomembranous colitis. A presumed diagnosis of Clostridium difficile colitis was made and no resection was performed. She was admitted to the surgical intensive care unit (ICU) with an ABThera ${ }^{\mathrm{TM}}$ (KCI USA, San Antonio, TX) abdominal vacuum dressing in place. She was treated

Department of Surgery, University of Michigan, Ann Arbor, Michigan.

(C) David Turer et al. 2016; Published by Mary Ann Liebert, Inc. This Open Access article is distributed under the terms of the Creative Commons License (http://creativecommons.org/licenses/by/4.0), which permits unrestricted use, distribution, and reproduction in any medium, provided the original work is properly credited. 

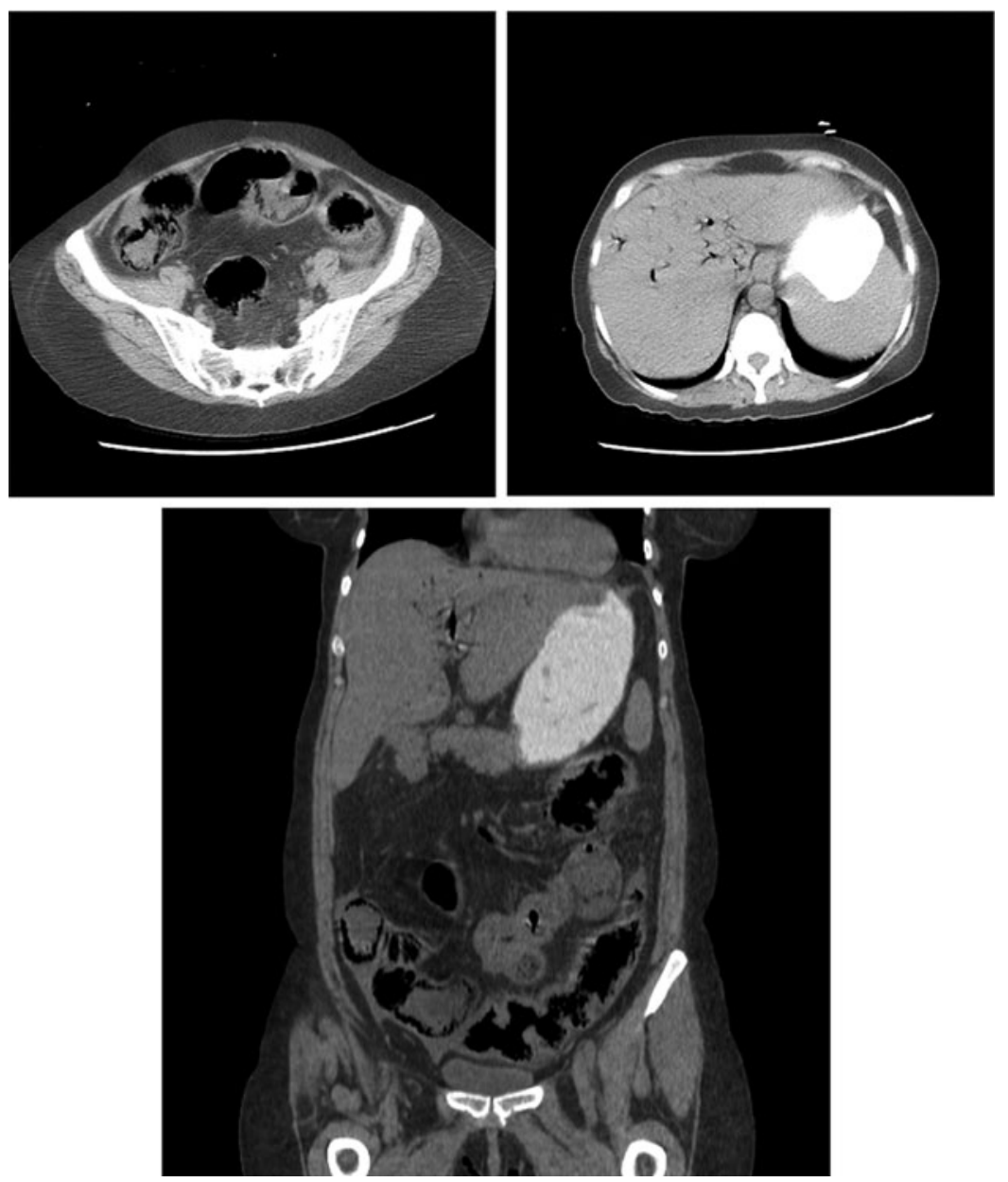

FIG. 1. Computed tomography (CT) scan obtained at the time of initial admission to the hospital revealed extensive pneumatosis along the wall of the distal small bowel and colon. Additional areas of mesenteric and portal venous gas were visualized.

with broad spectrum antibiotics as well as triple antibiotic therapy for $C$. difficile colitis (oral and rectal vancomycin and intravenous metronidazole). The patient was taken back to the operating room two days later for a planned second-look laparotomy. The surface of the small and large intestines appeared to have improved substantially from the time of the initial laparotomy; the abdomen was irrigated and the fascia was closed.

Post-operatively, the patient tested negative for $C$. difficile infection. However, her blood and abdominal fluid cultures were positive for Nocardia farcinica. On the basis of antibiotic sensitivity, imipenem-cilistatin, amikacin, and Bactrim $^{\circledR}$ (Hoffman-La Roche, Nutley, NJ) were added to her antibiotic regimen. Her post-operative course was complicated further by adrenal hemorrhage, diffuse pulmonary hemorrhage, acute respiratory distress syndrome (ARDS), and cytomegalovirus (CMV) viremia (although notably, her CMV counts were less than 300 copies per milliliter). She was found to be severely pancytopenic and a bone marrow biopsy revealed maturation arrest consistent with Nocardia infection. A subsequent flexible sigmoidoscopy on hospital day 16 revealed ulcerated lesions throughout the visualized colon that were biopsy negative for CMV. A repeat CT scan revealed no evidence of small bowel or colon ischemia. After a prolonged ICU admission, she was transitioned to a general care floor and was discharged to sub-acute rehabilitation.

The patient presented again to our institution three weeks after discharge with new onset right lower quadrant pain and sepsis. Computed tomography scan again revealed pancolitis with pneumatosis. Flexible sigmoidoscopy revealed edematous, friable mucosa with ulcerations, and biopsies were positive for severe CMV colitis. Cultures obtained by bronchoscopy also revealed CMV pneumonia. She was initiated on gancyclovir for treatment of CMV. After another prolonged ICU stay, she was eventually discharged back to subacute rehabilitation. After discharge, she was treated with a four-month course of oral valgancyclovir for CMV and a oneyear course of Bactrim for Nocardia.

\section{Conclusion}

We describe an unusual case of secondary infection with Nocardia in a patient who had been treated with a TNF- $\alpha$ antagonist for Crohn's disease. Nocardia infection is a rare but well-recognized side effect of tumor necrosis factor antagonists [1]. Furthermore, infection with Nocardia farcinica is also rare [2,3]. The presentation of Nocardia colitis observed in this patient is unusual and there has only been one 
case documented previously in the literature [4]. This case illustrates that patients being treated with TNF- $\alpha$ antagonists are susceptible to infections by atypical organisms. Patients with inflammatory bowel disease are often treated with multiple immunosuppressive medications and may be at risk for unusual infections, which should be included in the differential diagnosis when caring for this population.

\section{Author Disclosure Statement}

The authors have nothing to disclose.

\section{References}

1. Wallis RS, Broder MS, Wong JY, et al. Granulomatous infectious diseases associated with tumor necrosis factor antagonists. Clin Infect Dis 2004;38:1261-1265.

2. Torres $\mathrm{OH}$, Domingo $\mathrm{P}$, Pericas R, et al. Infection caused by Nocardia farcinica: Case report and review. Eur J Clin Microbiol Infect Dis 2000;19:205-212.

3. Al-Tawfiq JA, Al-Khatti AA. Disseminated systemic Nocardia farcinica infection complicating alefacept and infliximab therapy in a patient with severe psoriasis. Int $\mathbf{J}$ Infect Dis 2010;14:e153-e157.

4. Kamyab A, Fakhoury JDJ, Sutkowski R, et al. Fulminant colitis secondary to nocardiosis. Int J Colorectal Dis 2012; 27:841-842.
Address correspondence to: Dr. Krishnan Raghavendran Department of Surgery Division of Acute Care Surgery University of Michigan 1C-421 UH, SPC-5033 Ann Arbor, MI-48109

E-mail: kraghave@umich.edu

$$
\begin{aligned}
\text { Abbreviations Used } \\
\text { ARDS }=\text { acute respiratory distress syndrome } \\
\text { CMV }=\text { cytomegalovirus } \\
\mathrm{CT}=\text { computed tomography } \\
\mathrm{EGD}=\text { esophagogastroduodenoscopy } \\
\mathrm{ICU}=\text { intensive care unit } \\
\text { TNF- } \alpha=\text { tumor necrosis factor- } \alpha
\end{aligned}
$$

Cite this article as: Turer D, Gray B, Raghavendran K (2016) Fulminant nocardia colitis: A case report. Surgical Infections Case Reports 1:1, 69-71, DOI: 10.1089/ crsi.2016.0013. 